
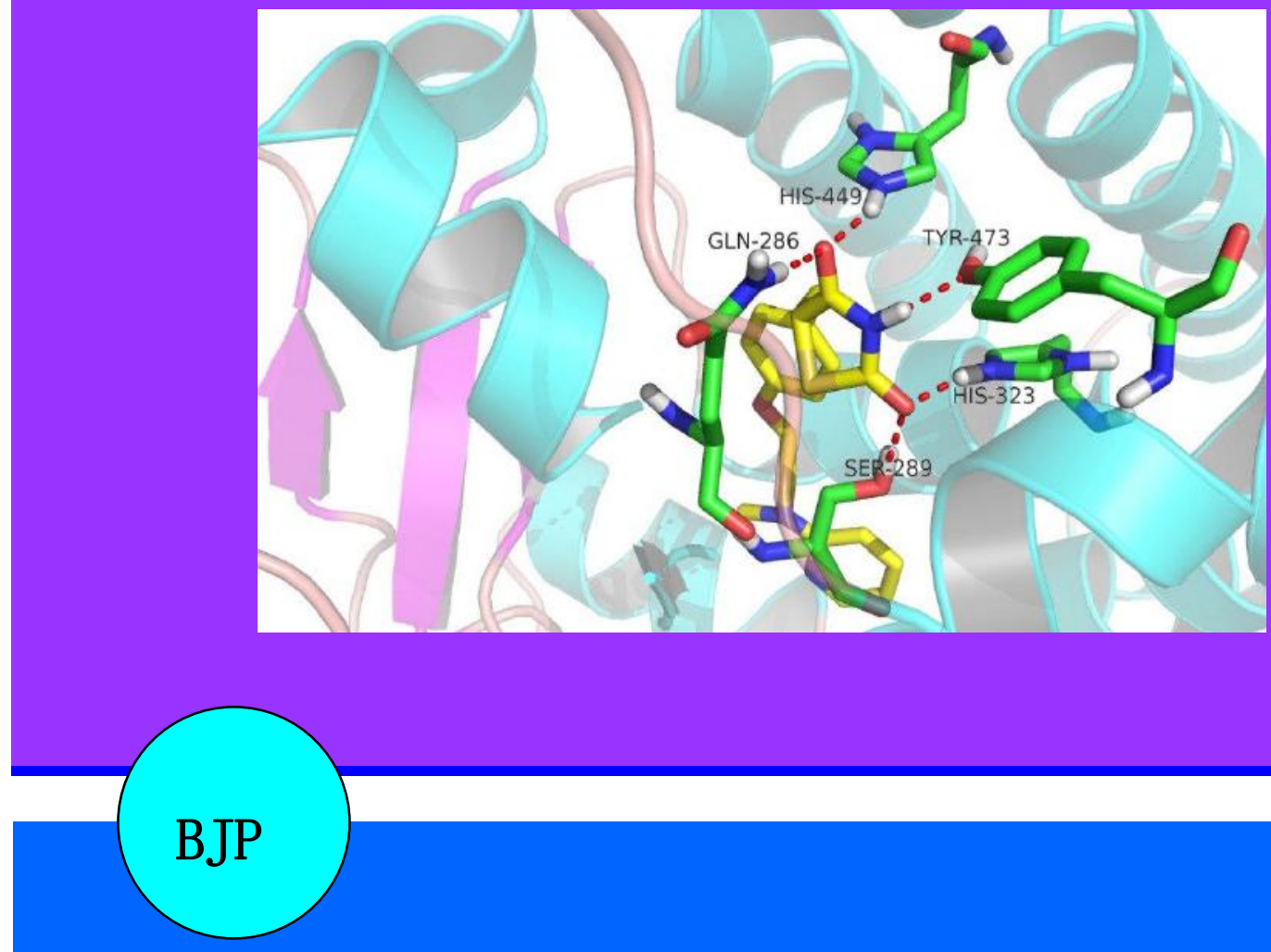

Bangladesh Journal of Pharmacology

Research Article

Molecular docking of citrus flavonoids with some targets related to diabetes 


\section{Molecular docking of citrus flavonoids with some targets related to diabetes}

\section{Wei Shen and Yan Hua Lu}

State Key Laboratory of Bioreactor Engineering, East China University of Science and Technology, Shanghai, PR China.

\begin{tabular}{|c|c|}
\hline \multicolumn{2}{|l|}{ Article Info } \\
\hline Received: & 16 March 2013 \\
\hline Accepted: & 22 March 2013 \\
\hline Available Online: & 19 April 2013 \\
\hline \multicolumn{2}{|c|}{ DOI: 10.3329/bjp.v8i2.14240 } \\
\hline \multicolumn{2}{|c|}{$\begin{array}{l}\text { Cite this article: } \\
\text { Shen W. Lu YH. Molecular docking of } \\
\text { citrus flavonoids with some targets } \\
\text { related to diabetes. Bangladesh J } \\
\text { Pharmacol. 2013; 8: 156-70. }\end{array}$} \\
\hline
\end{tabular}

\begin{abstract}
Citrus flavonoids isolated from citrus peel (flavedo and albedo) were taken as ligands for molecular docking. The molecular targets, (i.e. glucokinase, glycogen synthase kinases $3 \beta$, peroxisome proliferator-activated receptor gamma, and dipeptidyl peptidase IV) whose crystallographic structures are available on the PDB database as 1V4S, 1Q4L, 2PRG, 2ONC respectively, were used for the docking analysis using the Autodock tool V 4.2 and ADT v1.5.4 programs. The docking studies of the ligands citrus flavonoids with four different target proteins showed that citrus flavonoids are good molecules which dock well with various targets related to diabetes mellitus. The above results demonstrate that citrus flavonoids might be potentially used for blood glucose regulation.
\end{abstract}

\section{Introduction}

Diabetes mellitus has reached epidemic proportions and affects more than 170 million individuals worldwide (Stumvoll et al., 2005). Compared with synthetic compounds, natural small molecules with special bioactivity have become the major resource of bioactive agents and played a key role in diabetes therapy (Liu et al., 2010). Therefore, management of diabetes without any side effects is still a challenge to the medical system. This leads to increasing demand for natural products with antidiabetic activity with less side effects.

Citrus flavonoids have received much attention in recent years, for its potential therapeutic qualities and relatively low toxicity to animals (Benavente-Garcia and Castillo, 2008; Choi and Ahn, 2008; Manthey et al., 2001). In our previous study, hesperidin, naringin, neohesperidin and nobiletin exhibited antidiabetic activities partly by binding to starch, delaying the starch digestion (Shen et al., 2012). And these flavonoids showed week inhibitory activity against digestive enzymes (e.g., pancreatic a-amylase and a-glucosidase).
Likewise, hesperidin, naringin and nobiletin also showed hypoglycemic effects by improving insulin sensitivity in diabetic animals (Akiyama et al., 2009; Akiyama et al., 2010; Jung et al., 2006; Jung et al., 2004; Lee et al., 2010). However, whether these citrus flavonoids regulated blood glucose via other target proteins or genes related to diabetes were unclear.

Bioinformatics tools have become very important to pinpoint the targets for different ligands (Osguthorpe et al., 2012). Using bioinformatics tools we tried to evaluate whether citrus flavonoids are good ligands to some of the target proteins or gene related to diabetes such as glucokinase, glycogen synthase kinases $3 \beta$, peroxisome proliferator-activated receptor gamma, and dipeptidyl peptidase IV.

Glucokinase (GK, PDB ID: 1V4S) is a monomeric cytoplasmic enzyme found in the liver and pancreas. Its main function is regulation of glucose levels in these organs. Through phosphorylation glucokinase is able to increase the metabolism of glucose. In the liver it increases the synthesis of glycogen and it is the first 
step in glycolysis, the main producer of ATP in the body (Balamurugan et al., 2012; Kamata et al., 2004). Therefore, GK would be an ideal drug target for type 2 diabetes (T2D) diseases because of its high impact in glucose homeostasis, and its activation results in lower blood glucose level irrespective of the cause of hyperglycemia.

Glycogen synthase kinases $3 \beta$ (GSK 3 3 , PDB: 1Q4L) belongs to the super family of mitogen-activated protein kinase. GSK $3 \beta$ has been implicated in the development of insulin resistance and regulation of glycogen synthesis (Osolodkin et al., 011). It is one of the important targets in the treatment of T2D. Inhibitors of GSK-3 $\beta$ have antidiabetic properties because they improve insulin sensitivity, glycogen synthesis, and glucose metabolism in skeletal muscles of diabetic patients (Akhtar and Bharatam, 2012; Johnson et al., 2011; Khanfar et al., 2010; Wauwe, 2003).

Peroxisome proliferator-activated receptor gamma (PPARy, PDB: 2PRG) belongs to the nuclear receptor super family of transcription factors and is an important regulator of target genes involved in glucose and lipid homeostasis (Choi et al., 2011; Nolte et al., 1998). PPARY and their targets have been invested as attractive therapeutic targets for T2D (Bruning et al., 2007; Cho et al., 2011; Maltarollo and Honório, 2012).

Dipeptidyl peptidase IV (DPP IV, PDB: 2ONC) has become an attractive target of drug discovery and diabetes treatment (Drucker and Nauck, 2006; Verspohl, 2009). DPP IV is a membrane-bound, serine protease ectoenzyme found in numerous sites, including the kidney, intestine, and capillary endothelium. DPP IV is responsible for the degradation of a number of biological peptides including GLP-1 and GIP, which are incretins released from the gut in response to food and play an essential role in maintaining glucose homeostasis (Abu-Hamdah et al., 2009; Baggio and Drucker, 2007; Deacon, 2004; Verspohl, 2009).

The above mentioned targets were subjected to molecular docking with a view to identify how citrus flavonoids play an important role in the process of hyperglycemia.

\section{Methods and Materials}

\section{Retrieval of the three-dimensional structure of target proteins}

The structures of the target receptor binding sites of human glucokinase (PDB:1V4S), glycogen synthase kinases $3 \beta$ (PDB:1Q4L), peroxisome proliferator-activated receptor gamma (PDB:2PRG), and dipeptidyl peptidase IV (PDB:2ONC) were obtained from the
RCSB protein Data Bank, http://www.pdb.org/pdb/ home/home.do.

\section{Ligand selection}

Citrus flavonoids (i.e. hesperidin, naringin, neohesperidin, nobiletin) and other positive drugs were chosen from the National Centre for Biotechnology Informaton (NCBI) PubChem compound database. These molecules were downloaded in Structure Date File (SDF) format and converted to Protein Data Bank (PDB) coordinates by using Open Babel (http:// openbabel.org) converter. All the chemical structures of ligand compounds used in the study were shown in Figure 1.

\section{Receptor and ligand optimization}

PDB coordinates of the target receptor proteins and ligands molecules were optimized using Gromacs 4.0 suite force field analysis and UCSF Chimera (http:// www.cgl.ucsf.edu/chimera) tools, respectively. The optimized structures had minimum energy confirmation, which provided stability to the structure. These optimized receptors and ligands molecules were used for the docking study.

\section{Docking analysis}

The docking analysis of citrus flavonoids were carried out by means of the Autodock tools (ADT) v1.5.4 and autodock v4.2 program; (Autodock, Autogrid, Autotors, Copyright-1991e2000) from the Scripps Research Institute, http://www.scripps.edu/mb/ olson/doc/autodock. To run autodock, we used a searching grid extended over ligand moieties, Kollman charges were assigned and atomic solvation parameteres were added. Polar hydrogen charges of the Gasteiger-type were assigned and nonpolar hydrogens were merged with the carbons and the internal degrees of freedom and torsions were set. Citrus flavonoids were docked to all the target protein complexes with the molecule considered as a rigid body. The search was extended over the whole receptor protein used as blind docking. Affinity maps for all the atom types present, as well as an electrostatic map, were computed with a grid spacing of 0.375 . The search was carried out with the Lamarckian Genetic Algorithm; populations of 100 individuals with a mutation rate of 0.02 have been evolved for 10 generations. The remaining parameters were set as default. A root mean square deviation (RMSD) tolerance for each docking was set at 2.0. Evaluation of the results was done by sorting the different complexes with respect to the predicted binding energy. A cluster analysis based on root mean square deviation values, with reference to the starting geometry, was subsequently performed and the lowest energy conformation of the more populated cluster was 


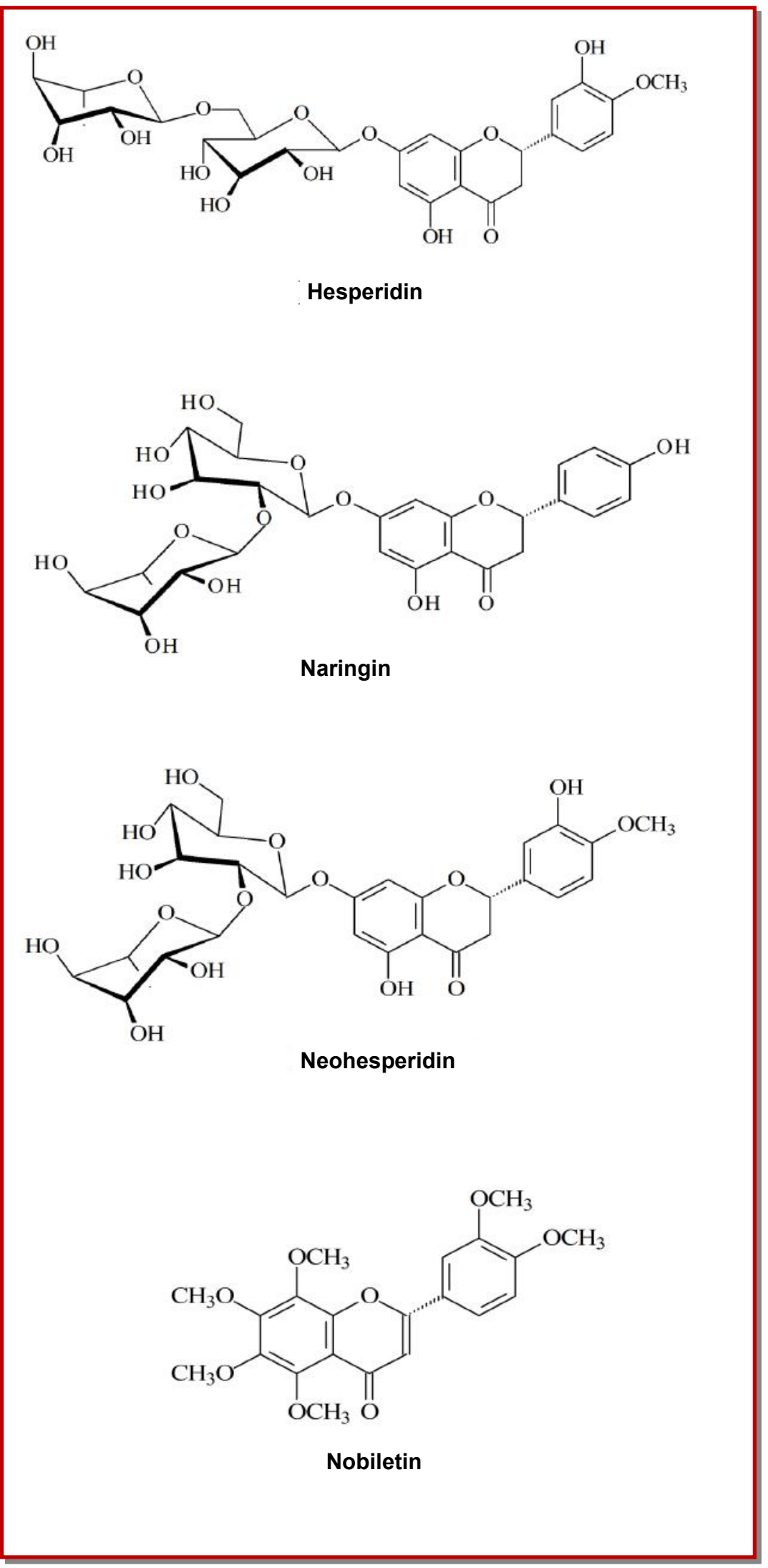

Figure 1: Structures of complexes used in this study 


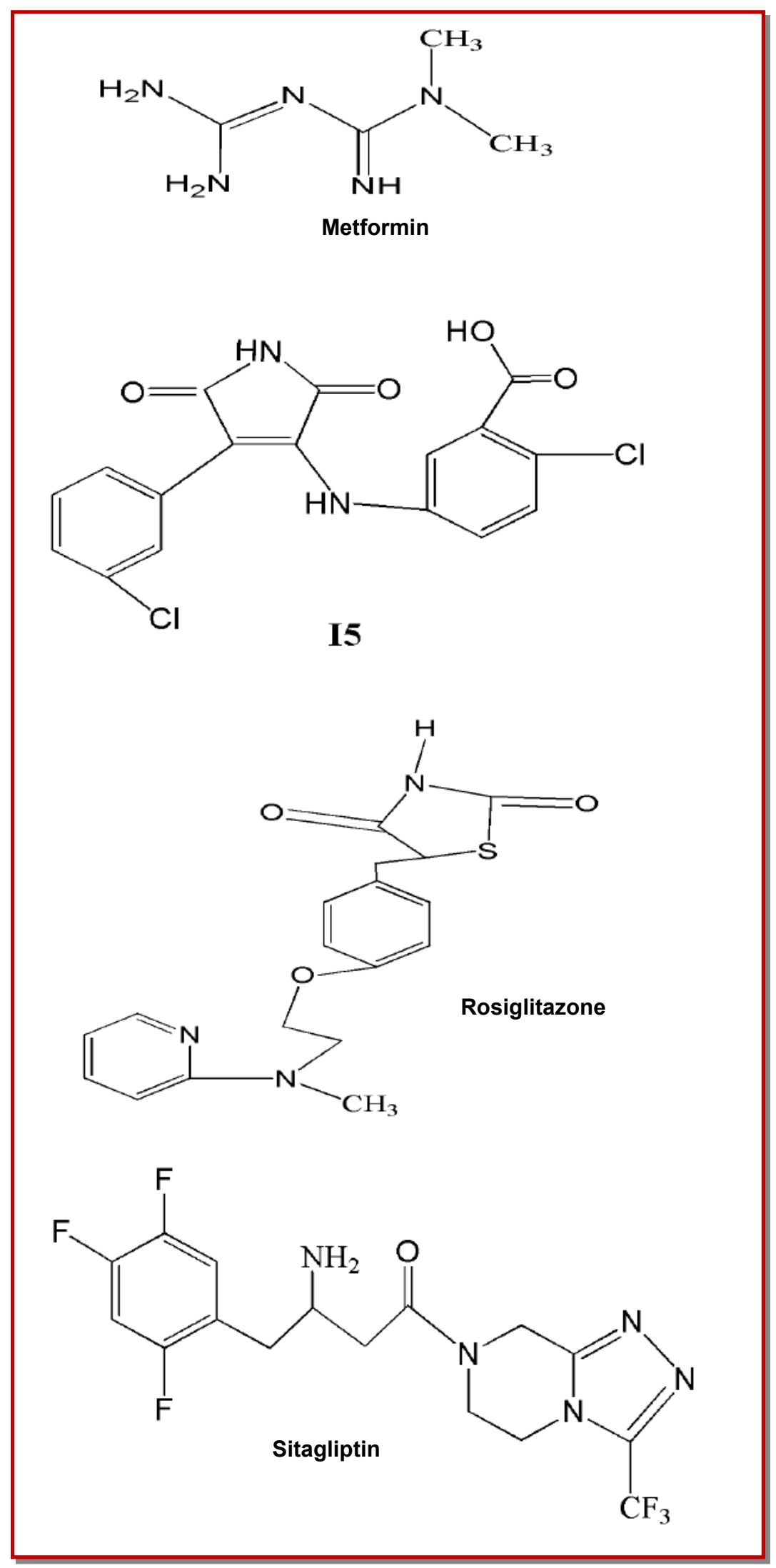

Figure 1: Structures of complexes used in this study (Cont.) 
considered as the most reliable solution.

\section{Results and Discussion}

The docking simulations in the active sites of 1V4S, 1Q4L, 2PRG, and 2ONC were performed by the Auto dock program, which has been shown to successfully reproduce experimentally observed binding modes in terms of lowest docking energy. The target proteins structures of 1V4S, 1Q4L, 2PRG, and 2ONC were docked with citrus flavonoids, which provided excellent results as were seen by the least values of the binding energy.

The best possible binding modes of all the ligands at target protein's active sites are displayed in Figure 2-5 by using PYMOL tool v1.1. Ligands hydrogen-bonding to four target proteins and their corresponding energy values are listed in Table I-IV.

Figure 2 shows the result of docking analysis of human glucokinase (1V4S) with citrus flavonoids. Figure 2A showed the binding site of protein and metformin. Glucokinase protein residues Glu 216, Tyr 215, Glu 96 were formed $\mathrm{H}$-bond with metformin. Figure 2B showed that hesperidin exhibited strong interaction with glucokinase protein. It formed $9 \mathrm{H}$-bonds i.e. Arg 63, Thr 65, Tyr 214, Tyr 215, Val 452, Cys 220. Figure 2C illustrated that naringin also exhibited strong interaction with 1V4S via forming H-bonds with Arg 63, Pro 66, Val 452, Val 455, Ieu 451, Asp 158 residues. Figure 2D showed that nobiletin formed H-bonds with Thr 65, Tyr 215, Leu 451 residues. Figure 2E depicted that neohesperidin exhibited weak interaction with glucokinase via H-bond interaction with Ser 64, Tyr 215 residues. The glucokinase resultant binding energy, $K_{i}$, $\mathrm{H}$-bond and other interaction with the active site residues are given below in the Table I.

Glucokinase is expressed only in liver and pancreatic beta cells and plays a key role in the regulation of glucose homeostasis. In the hepatocyte, the phosphorylation of glucose by glucokinase facilitates the uptake and metabolism of glucose by maintaining a gradient for glucose transport into these cells thereby regulating hepatic glucose disposal. In the beta cells, GK is believed to be part of the glucose-sensing mechanism and to be involved in the regulation of insulin release (Stoffel et al., 1992). During diabetes condition total or partial deficiency of insulin causes derangements in carbohydrate metabolism that decreases activity of several key enzymes including glucokinase resulting in the impaired glucose utilization and augmented hepatic glucose production. Chandramohan et al. reported that diabetic rats treated with 3-HMX active principle from plant increased GK activity (Chandramohan et al., 2008). In the same way citrus flavonoids increases GK activity by docking into GK's active sites, thereby increasing the utilization of glucose leading to decreased blood glucose level.

Figure 3A showed binding interaction of protein-I5 via Val 135, Asp 133, Gln 185, Arg 141 residues. Figure 3B exhibited binding interaction of protein-hesperidin via forming H-bonds with Ile 62, Val 135, Gln 185, Lys 183, Arg 141, Asp 200 residues. Figure 3C illustrated that naringin exhibited strong interaction with GSK-3 $\beta$ protein via forming H-bond with Lys 85, Ile 62, Gln 185, Glu 97, Arg 141, Tyr 134, Pro 136, Val 135, Asp 133 residues. Figure 3D depicted the binding interaction of protein-neohesperidin via Ile 62, Arg 141, Val 135, Gln 185, Asp 133, Asp 200 residues. Figure 3E showed the interaction between GSK- $3 \beta$ and nobiletin. Two Hbonds were formed between GSK- $3 \beta$ and nobiletin. The GSK- $3 \beta$ resultant binding energy, $K_{i}, \mathrm{H}$-bond and other interaction with the active site residues are given below in the Table II.

GSK-3 $\beta$ can be inhibited through three distinct mechanisms: i), ATP non-competitive (in substrate interaction domain), ii), ATP competitive (in ATP binding pocket), and iii) metal ion competitive (in $\mathrm{Mg} 2+$ binding site). GSK-3 $\beta$ has been identified as an important kinase in the intercellular signaling pathway downstream from the insulin receptor. GSK-3 $\beta$ inactivates GS by phosphorylation resulting in glycogen synthesis inhibition (Khanfar et al., 2010; Wauwe, 2003). It has been reported that some flavonoids, such as luteolin, rutin, narirutin, etc., could bind with B-ring hydroxyls stabilized by hydrogen bonding with Arg 141 and Tyr 134 in the hinge; A-ring hydroxyls stabilized by hydrogen bonding with Asn 64, Gly 65, Lys 85, and Asp 200 residues in the glycine-rich loop (Johnson et al., 2011). Our results indicated that four citrus flavonoids could form H-bonds or via other interactions with the above amino acid residues. Therefore, citrus flavonoids showed GSK-3 $\beta$ inhibitory activity. Moreover, among the four tested citrus flavonoids, hesperidin and naringin present the better inhibitory activity against GSK-3 $\beta$.

Figure 4 depicts the docking analysis of PPARy. Rosiglitazone is full agonists of PPAR . Figure 4A showed the hydrogen-bonding network of the rosiglitazone head group to conserved PPARY residues. Figure 4B showed the hydrogen-bonding network of the hesperidin to protein residues Tyr 327, Tyr 473, Ser 342, Glu 291, His 449, Ile 326, Arg 288. Figure 4C depicted the orientation of naringin bound in the active site of the PPAR $\gamma$ crystal structure. Figure 4D illustrated hydrogen-bonding interaction of nobiletin to PPARY protein Arg 288, Glu 343 residues. Figure $4 \mathrm{E}$ showed the neohesperidin formed H-bonds with protein residues Leu 340, Glu 291, Gly 284. The PPARY resultant binding energy, $K_{i}$, H-bond and other interaction with the active site 


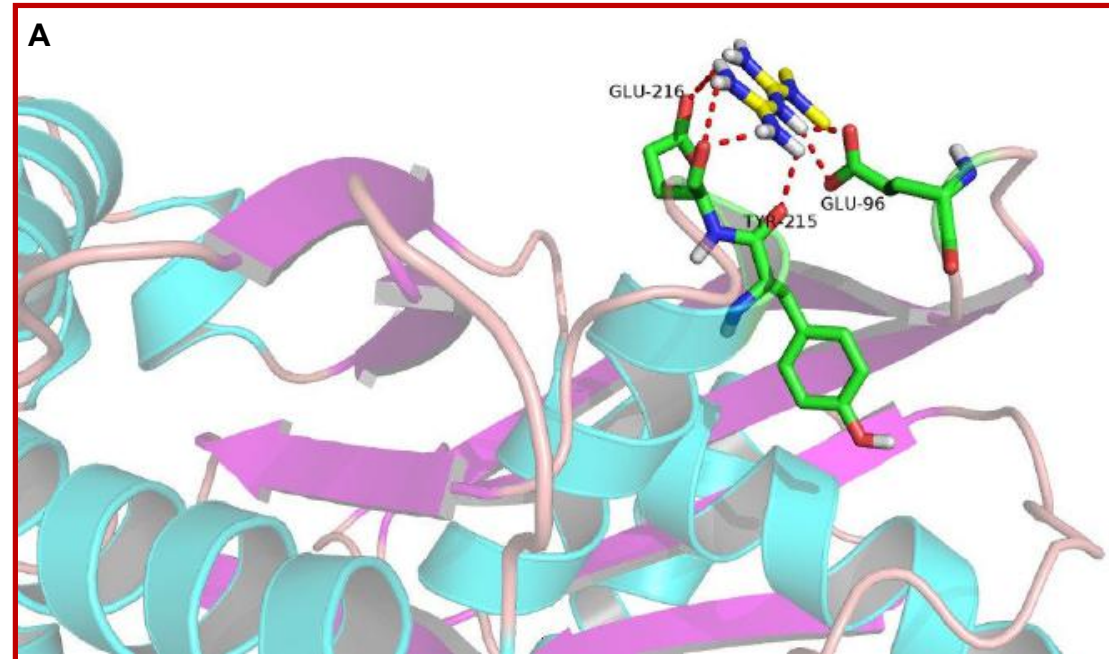

B
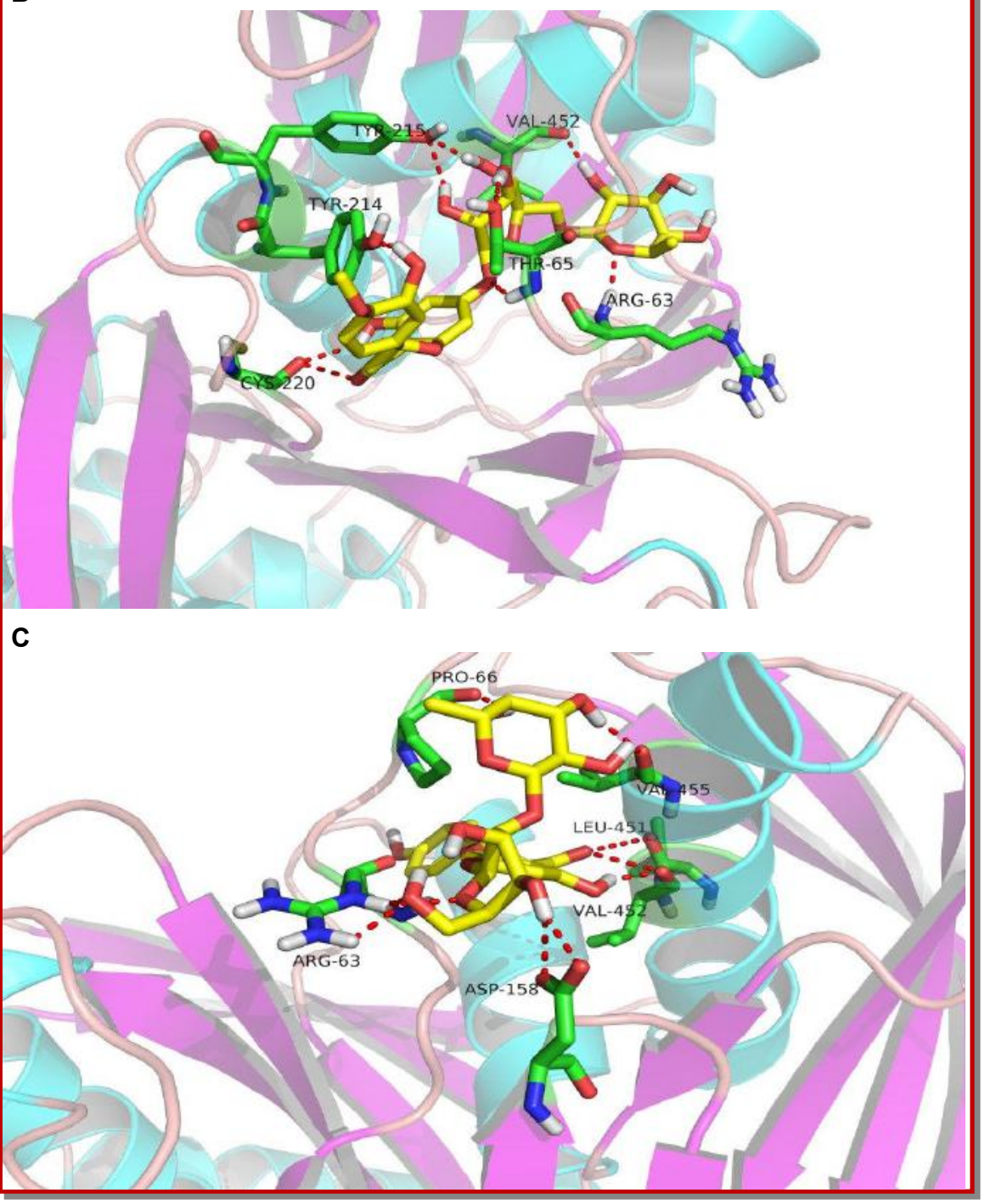

Figure 2: Docking model predicted structural details of hydrogen-bonding networks of metformin and citrus flavonoids. (A), (B), (C), (D) and (E) are represent for hydrogen-bonding network of metformin, hesperidin, naringin, nobiletin and neohesperidin to GK (PDB code: $1 \mathrm{~V} 4 \mathrm{~S})$ residues, respectively 


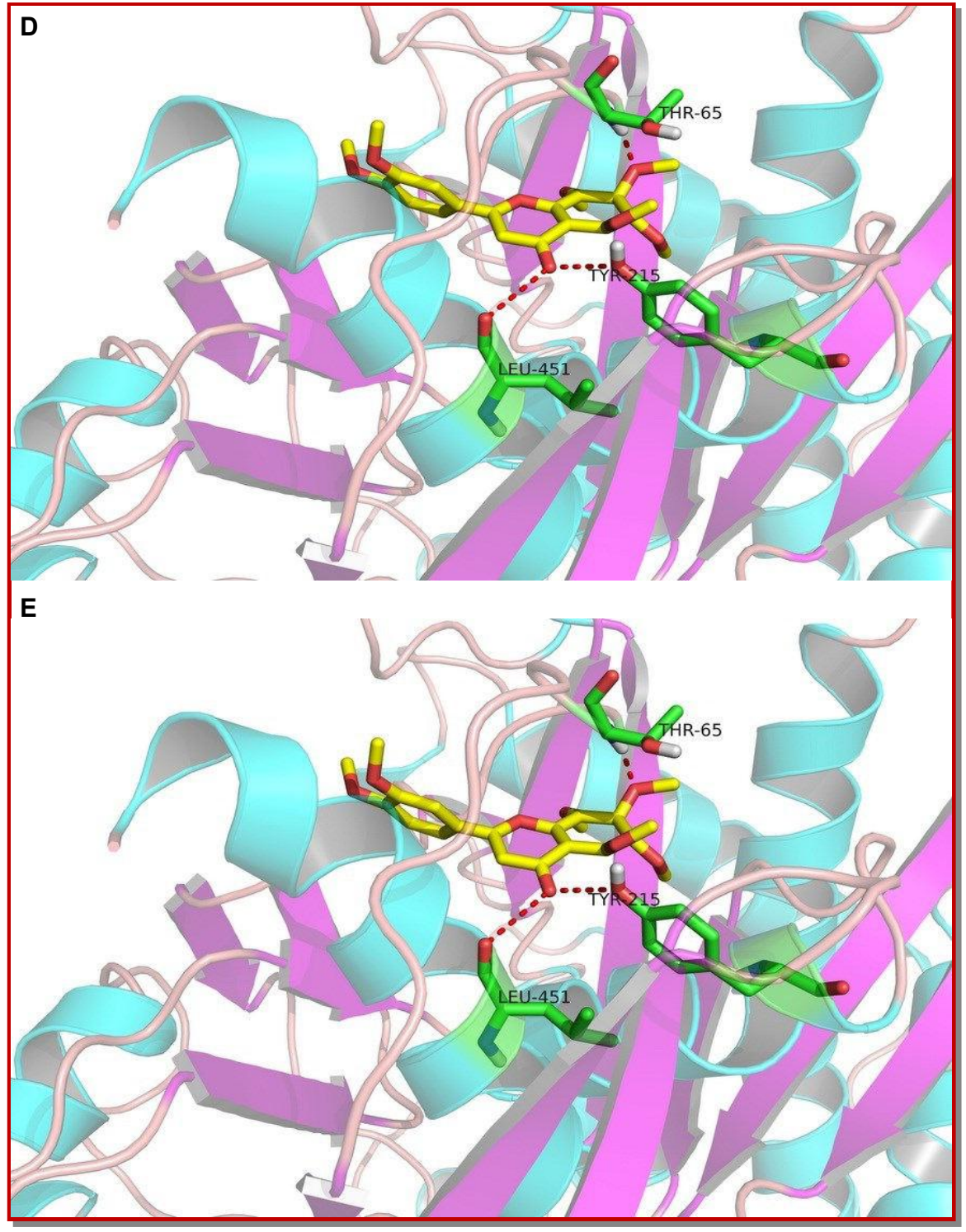

Figure 2: Docking model predicted structural details of hydrogen-bonding networks of metformin and citrus flavonoids. (A), (B), (C), (D) and (E) are represent for hydrogen-bonding network of metformin, hesperidin, naringin, nobiletin and neohesperidin to GK (PDB code: 1V4S) residues, respectively (Cont.)

\begin{tabular}{|c|c|c|c|}
\hline \multicolumn{4}{|c|}{ Table I } \\
\hline \multicolumn{4}{|c|}{ Hit list the interacting residues, binding energy and inhibit contant of docked ligands to GK receptor } \\
\hline & Interacting amino acid residues & $\begin{array}{l}\text { Binding energy } \\
(\mathrm{kcal} / \mathrm{mol})\end{array}$ & $\mathrm{K}_{\mathrm{i}}(\mathrm{nM})$ \\
\hline Metformin & GLU 96, TYR 215, GLU 216. & -7.9 & 1700 \\
\hline Hesperidin & $\begin{array}{l}\text { ARG 63, THR 65, TYR 214, TYR 215, VAL 452,CYS 220. HIS 218, ILE 211, ALA } \\
\text { 456, MET 235, SER 64, VAL 62, PRO 66, GLU } 221 .\end{array}$ & -10.8 & 13.2 \\
\hline Naringin & $\begin{array}{c}\text { ARG 63, PRO 66, VAL 452, VAL 455, IEU 451, ASP 158. LYS 459, ALA 456, } \\
\text { THR 65, TYR 214, TYR } 61 .\end{array}$ & -10.4 & 23.4 \\
\hline Neohesperidin & $\begin{array}{l}\text { SER 64, TRY 215. GLY 249, TYR 214, VAL 455, ALA 456, ILE 159, VAL452, } \\
\text { ARG 63, VAL 62, PRO 66, THR } 65 .\end{array}$ & -7.3 & 4430 \\
\hline Nobiletin & $\begin{array}{l}\text { THR 65, TYR 215, LEU 451. ALA 456, VAL 455, VAL 452, TYR 214, ILE 211, } \\
\text { MET 210, VAL 62, THR 61, ILE 159, MET 235, ARG } 63 .\end{array}$ & -9.4 & 134.6 \\
\hline
\end{tabular}




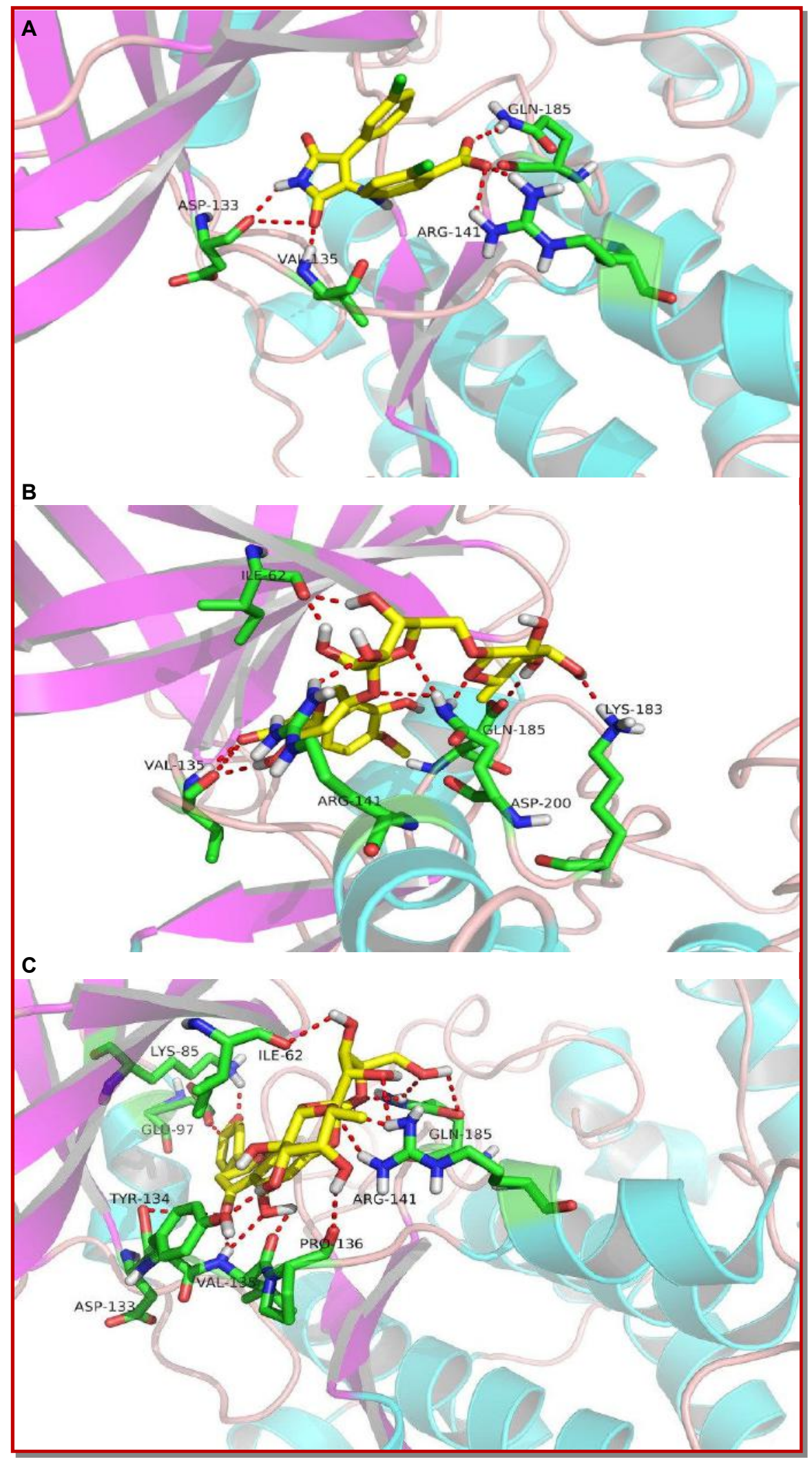

Figure 3: Docking model predicted structural details of hydrogen-bonding networks of $I 5$ and citrus flavonoids. (A), (B), (C), (D) and (E) are represent for hydrogen-bonding network of I5, hesperidin, naringin, neohesperidin and nobiletin to GSK-3 $\beta$ (PDB code: $1 \mathrm{Q} 4 \mathrm{~L}$ ) residues, respectively 


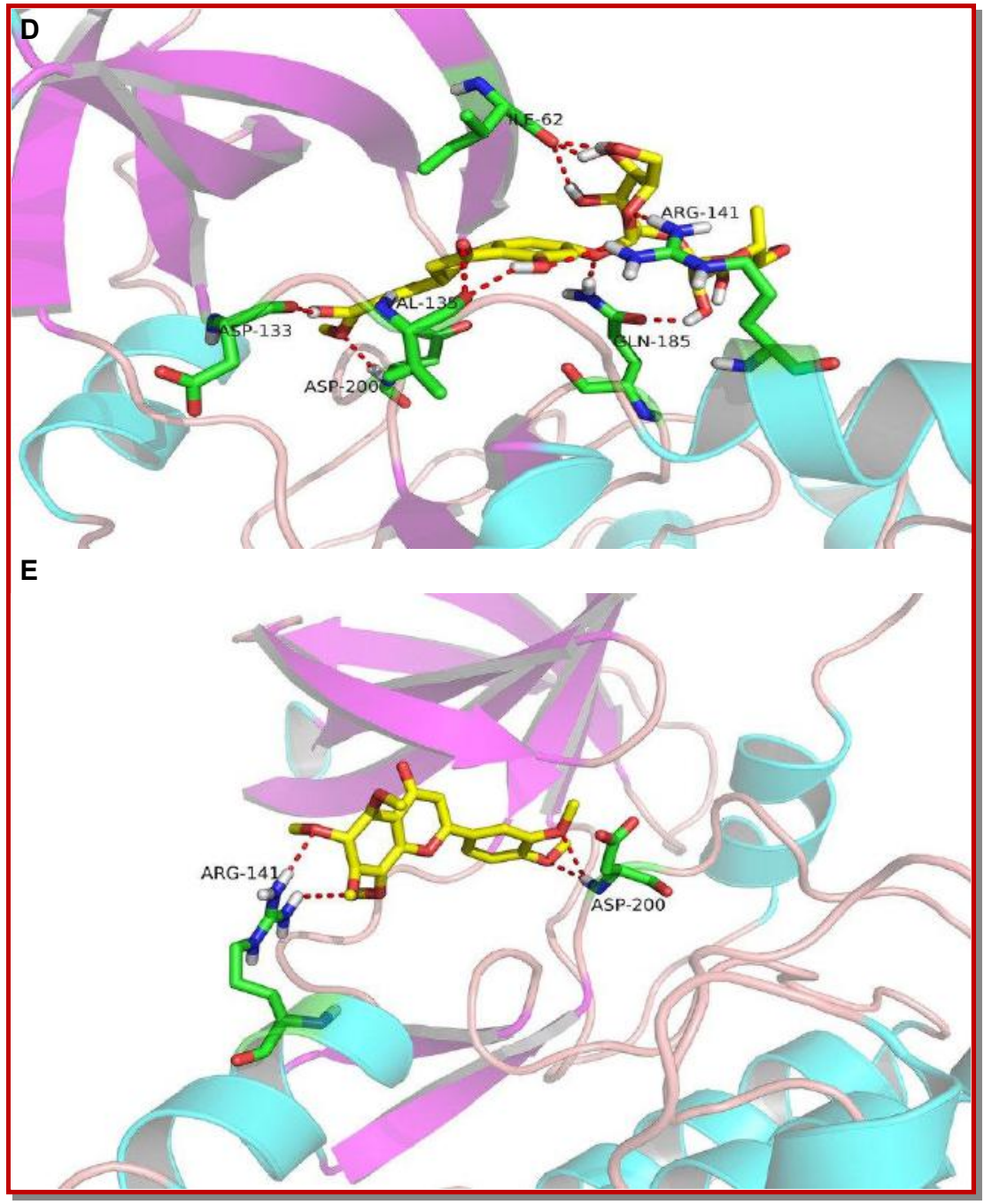

Figure 3: Docking model predicted structural details of hydrogen-bonding networks of I5 and citrus flavonoids. (A), (B), (C), (D) and $(\mathrm{E})$ are represent for hydrogen-bonding network of $\mathrm{I} 5$, hesperidin, naringin, neohesperidin and nobiletin to GSK-3 $\beta$ (PDB code: 1Q4L) residues, respectively (Cont.)

\section{Table II}

Hit list the interacting residues, binding energy and inhibit contant of docked ligands to GSK-3 $\beta$ receptor

\begin{tabular}{|c|c|c|c|}
\hline & Interacting amino acid residues & $\begin{array}{l}\text { Binding energy } \\
(\mathrm{kcal} / \mathrm{mol})\end{array}$ & $\mathrm{K}_{\mathrm{i}}(\mathrm{nM})$ \\
\hline I5 & $\begin{array}{l}\text { VAL 135, ASP 133, GLN 185, ARG 141. MET 101, GLU 97, LYS 85, ALA 83, } \\
\text { VAL 70, GLY 63, SER 66, LEU 132, ILE 62, PHE 201, ASP 200, VAL 110, } \\
\text { CYS 199, ASN 186, LYS 183, LEU 188, THR } 138 .\end{array}$ & -8.9 & 278.4 \\
\hline Hesperidin & $\begin{array}{l}\text { ILE 62, VAL 135, GLN 185, LYS 183, ARG 141, ASP 200. ALA 83, LEU 132, } \\
\text { LYS 85, MET 101, GLU 97, VAL 70, VAL 110, PHE 201, CYS 199, SER 66, } \\
\text { ASN 186, THR 138, LYS 183, ASN 64, GLY 63, LEU 188. }\end{array}$ & -9.8 & 64.4 \\
\hline Naringin & $\begin{array}{c}\text { LYS 85, ILE 62, GLN 185, GLU 97, ARG 141, TYR 134, PRO 136, VAL 135, } \\
\text { ASP 133. LEU 132, ALA 88, LEU 188, THR 138, CYS 199, ASP 200, PHE } \\
\text { 201, ALA 83, GLY 63. }\end{array}$ & -9.7 & 74.0 \\
\hline Neohesperidin & $\begin{array}{l}\text { ILE 62, ARG 141, VAL 135, GLN 185, ASP 133, ASP 200. LEU 132, VAL } \\
\text { 110, LEU 188, TYR 134, GLY 63, THR 138, TYR } 140 .\end{array}$ & -8.3 & 826.3 \\
\hline Nobiletin & $\begin{array}{l}\text { ARG 141, ASP 200. LYS 85, GLY 63, ILE 62, IEU 132, CYS 199, GLN 185, } \\
\text { THR } 138 .\end{array}$ & -7.0 & 7080 \\
\hline
\end{tabular}




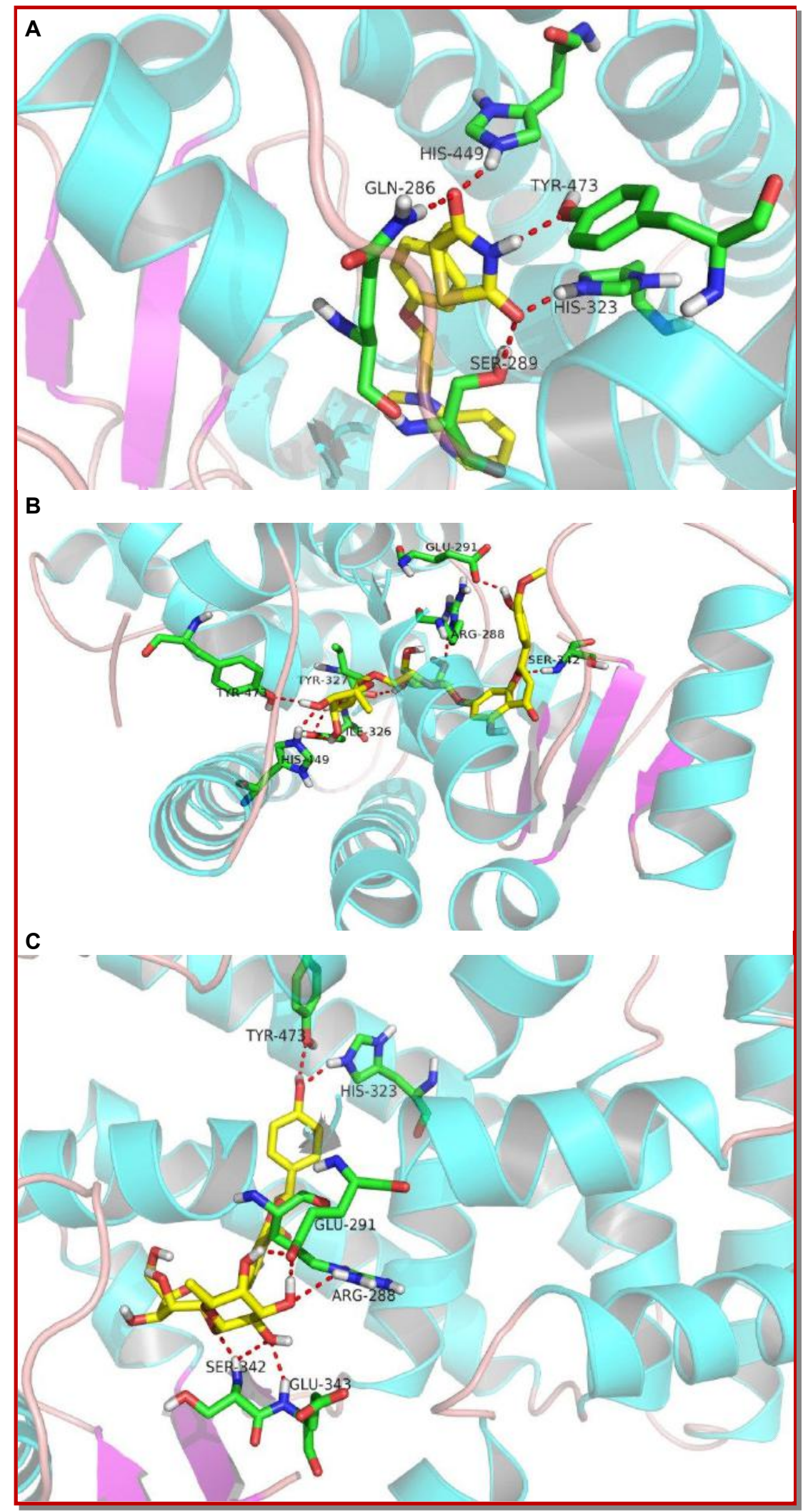

Figure 4: Docking model predicted structural details of hydrogen-bonding networks of rosiglitazone and citrus flavonoids. (A), (B), (C), (D) and (E) are represent for hydrogen-bonding network of rosiglitazone, hesperidin, naringin, nobiletin and neohesperidin to PPAR Y (PDB code: 2 PRG) residues, respectively 


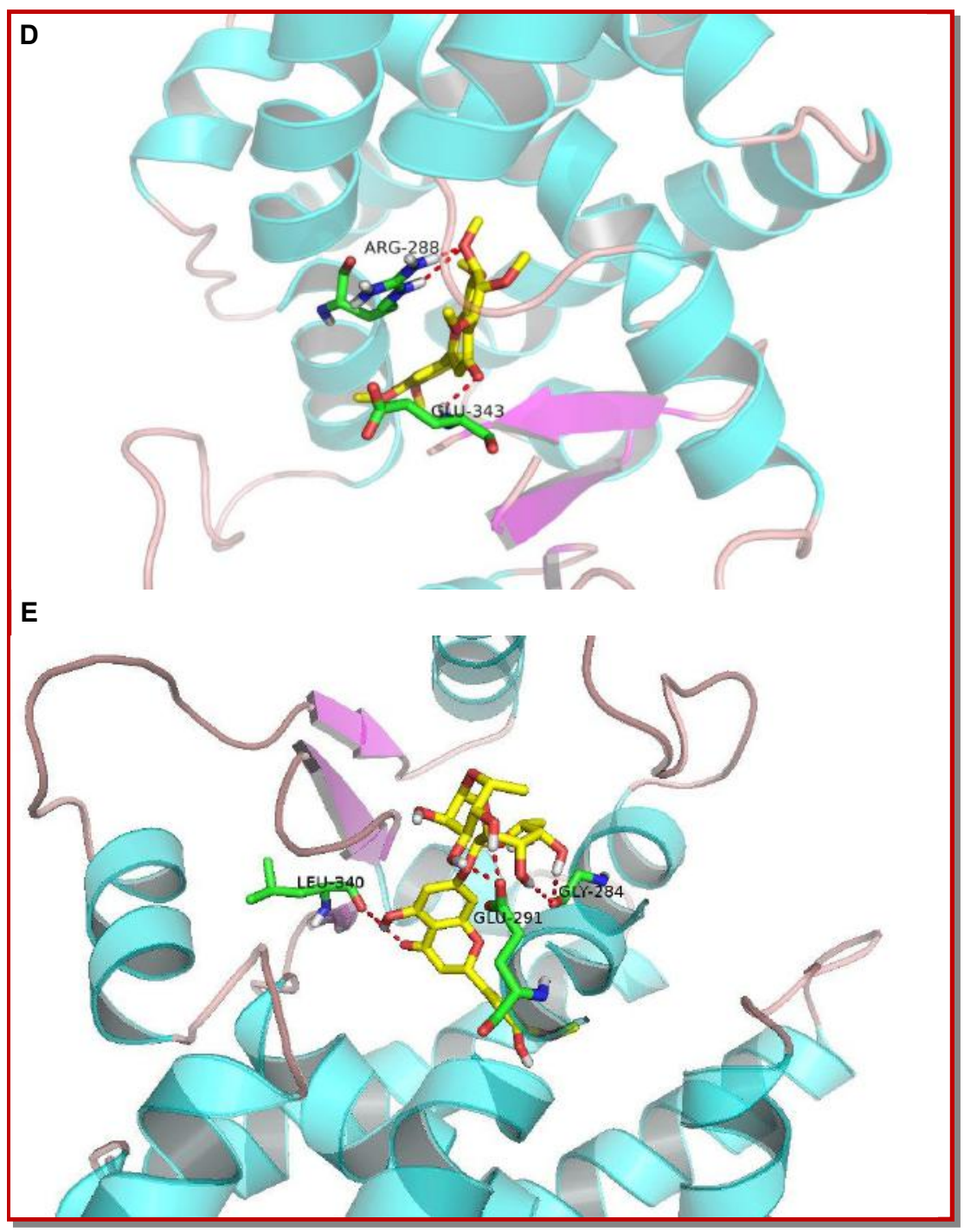

Figure 4: Docking model predicted structural details of hydrogen-bonding networks of rosiglitazone and citrus flavonoids. (A), (B), (C), (D) and (E) are represent for hydrogen-bonding network of rosiglitazone, hesperidin, naringin, nobiletin and neohesperidin to PPAR Y (PDB code: 2PRG) residues, respectively (Cont.)

\section{Table III}

Hit list the interacting residues, binding energy and inhibit contant of docked ligands to PPAR Y receptor

\begin{tabular}{|c|c|c|c|}
\hline & Interacting amino acid residues & $\begin{array}{l}\text { Binding energy } \\
(\mathrm{kcal} / \mathrm{mol})\end{array}$ & $\mathrm{K}_{\mathrm{i}}(\mathrm{nM})$ \\
\hline Rosiglitazone & $\begin{array}{c}\text { GLN 286, TYR 473, SER 289, HIS 323, HIS 449. ARG 288, CYS 285, LEU 330, } \\
\text { MET 329, ILE 326, ALA 292, LEU } 469 .\end{array}$ & -10.5 & 21.4 \\
\hline Hesperidin & $\begin{array}{l}\text { TYR 327, TYR 473, SER 342, GLU 291, HIS 449, ILE 326, ARG 288. CYS 285, } \\
\text { LEU 330, SER 289, GLN 286, ILE341, PHE } 287 .\end{array}$ & -9.6 & 89.8 \\
\hline Naringin & $\begin{array}{l}\text { TYR 473, HIS 323, ARG 288, GLU 291, SER 342, GLU 343. ILE 341, LEU 330, } \\
\text { GLN 286, MET 364, CYS 285, GLY 284, SER 289, LEU 270, ILE 326, PHE } 287 .\end{array}$ & -8.7 & 436.2 \\
\hline Neohesperidin & $\begin{array}{l}\text { LEU 340, GLU 291, GLY 284. GLN 286, SER 289, CYS 285, LEU 330, ILE 341, } \\
\text { ARG288, SER 342, GLU 343, VAL339, THR 268, LEU 270, PHE } 287 .\end{array}$ & -8.0 & 1370 \\
\hline Nobiletin & $\begin{array}{l}\text { ARG 288, GLU 343. CYS 285, GLY 284, ILE 341, MET 329, LEU 330, SER 342, } \\
\text { LEU 228, LEU } 333 .\end{array}$ & -8.2 & 941.9 \\
\hline
\end{tabular}




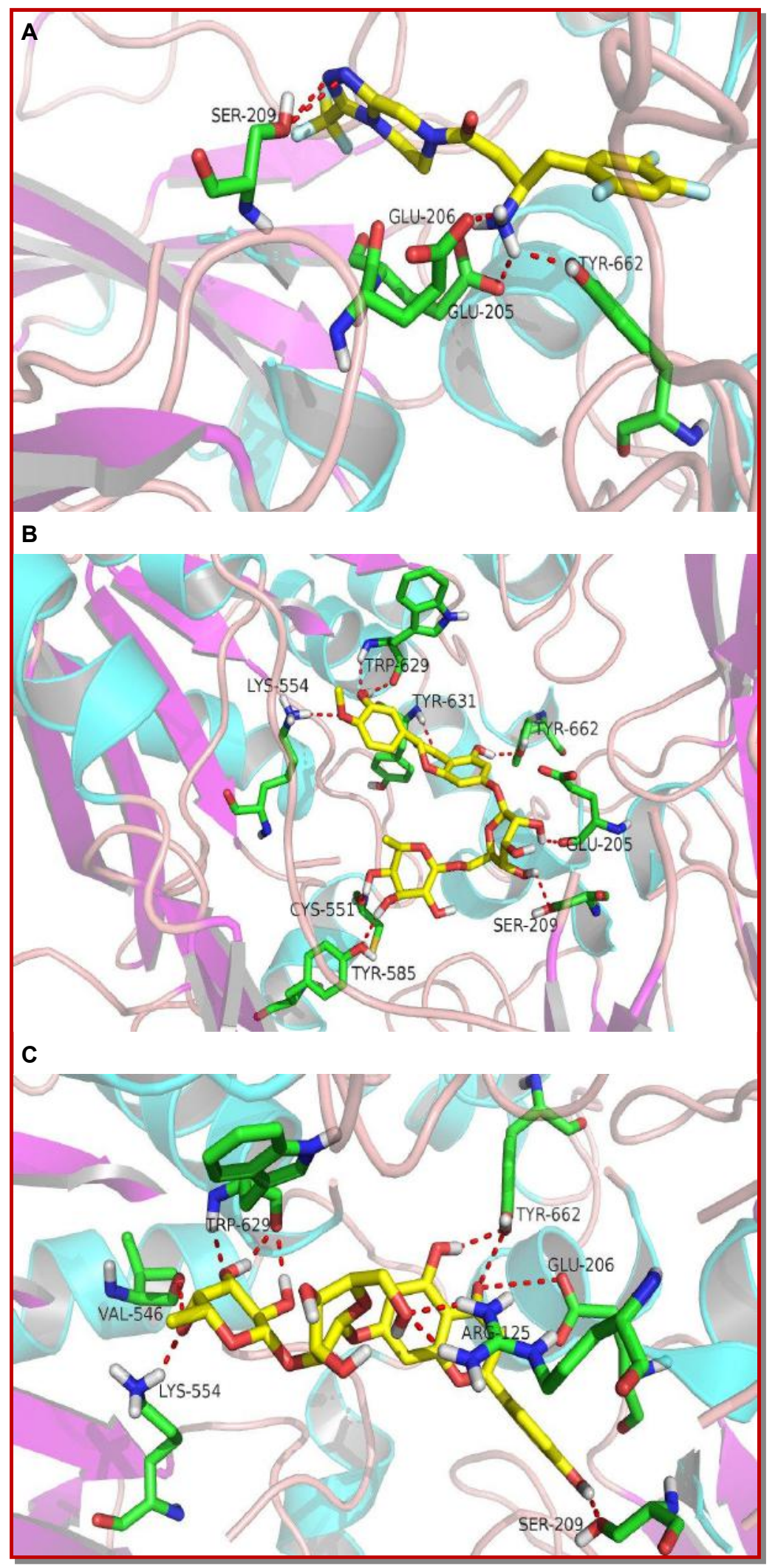

Figure 5: Docking model predicted structural details of hydrogen-bonding networks of sitagliptin and citrus flavonoids. (A), (B), (C), (D) and (E) are represent for hydrogen-bonding network of sitagliptin, hesperidin, naringin, neohesperidin and nobiletin to DPPIV(PDB code: 2ONC) residues, respectively 


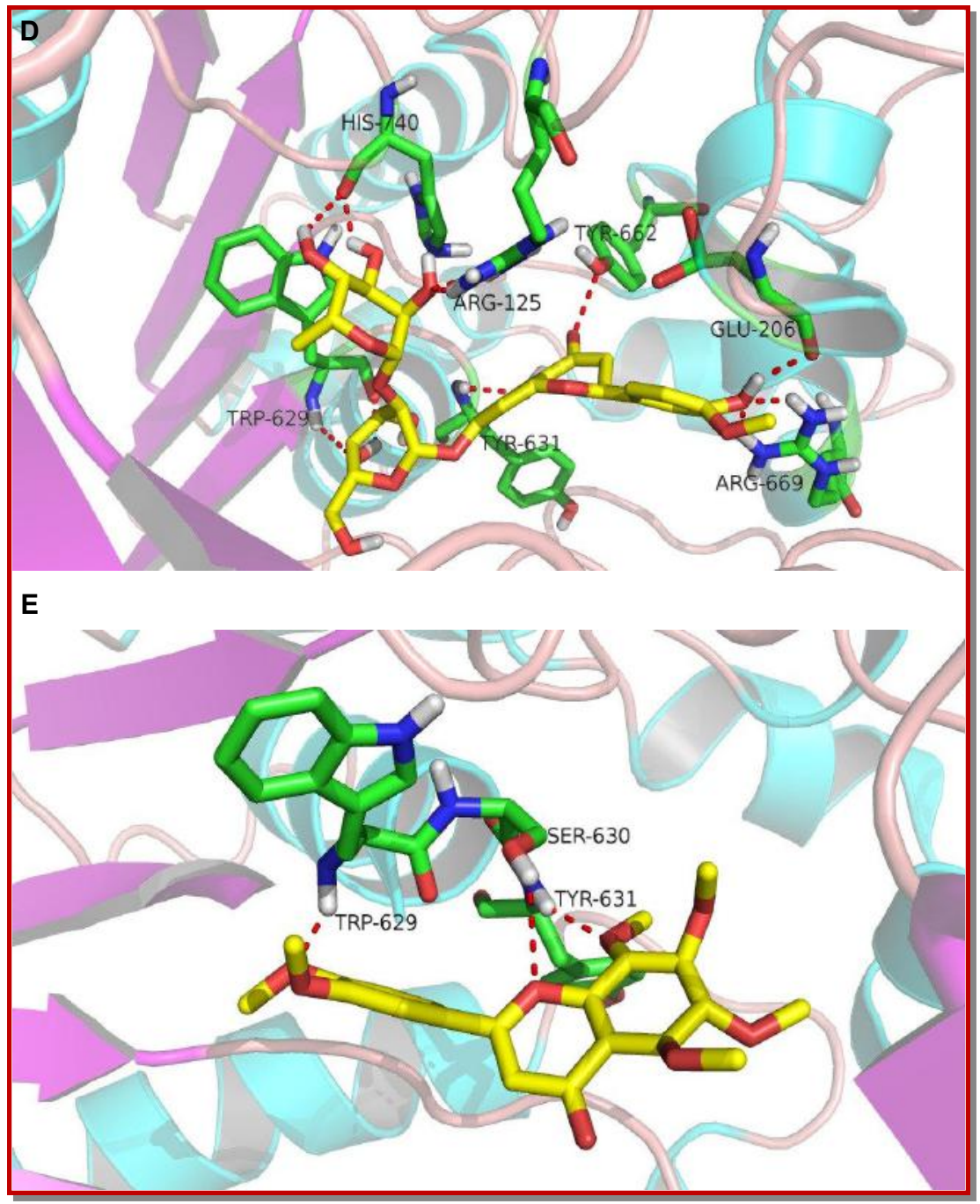

Figure 5: Docking model predicted structural details of hydrogen-bonding networks of sitagliptin and citrus flavonoids. (A), (B), (C), (D) and (E) are represent for hydrogen-bonding network of sitagliptin, hesperidin, naringin, neohesperidin and nobiletin to DPPIV(PDB code: $2 \mathrm{ONC}$ ) residues, respectively (Cont.)

Table IV

Hit list the interacting residues, binding energy and inhibit contant of docked ligands to DPPIV receptor

\begin{tabular}{|c|c|c|c|}
\hline & Interacting amino acid residues & $\begin{array}{l}\text { Binding energy } \\
(\mathrm{kcal} / \mathrm{mol})\end{array}$ & $\mathrm{K}_{\mathrm{i}}(\mathrm{nM})$ \\
\hline Sitagliptin & $\begin{array}{c}\text { GLU 205, GLU 206, TYR 662, SER 209. TYR 361, VAL 656, SER 630, VAL } \\
\text { 711, HIS 740, TYR 666, PHE 357, ARG } 358 .\end{array}$ & -9.3 & 155.2 \\
\hline Hesperidin & $\begin{array}{l}\text { GLU 205, SER 209, CYS 551, LYS 554, TYR 585, TRP 629, TYR 631, TYR } \\
\text { 662. TYR 666, PHE 357, GLU 206, ARG 125, HIS 740, TRP 547, SER 630, } \\
\text { GLY 632, VAL 546. }\end{array}$ & -8.3 & 782.2 \\
\hline Naringin & $\begin{array}{l}\text { VAL 546, SER 209, TYR 662, TRP 629, GLU 206, ARG 125, LYS 554. TYR } \\
\text { 666, PHE 357, GLU 205, HIS 740, TYR 547, SER 630, GLY } 632 .\end{array}$ & -8.4 & 725.8 \\
\hline Neohesperidin & $\begin{array}{l}\text { GLU 206, HIS 740, TRP 629, ARG 669, ARG 125, TYR 631, TRP 662. SER } \\
\text { 630, GLY 631, TYR 547, TYR 666, PHE 357, GLU 206, GLY 741, LYS } 554\end{array}$ & -8.2 & 978.3 \\
\hline Nobiletin & $\begin{array}{l}\text { TRP 629, SER 630, TYR 631. TYR 547, TYR 662, TYR 666, GLU 206, ARG } \\
\text { 125, TRP 629, ASN 710, ASP 545, VAL 546, LYS } 554 .\end{array}$ & -6.7 & 13310 \\
\hline
\end{tabular}


residues are given below in the Table III.

It has been reported that rosiglitazone could form hydrogen bonds with residues His 323, Tyr 473, His 449, Ser 289, Gln286, Tyr 327 of PPARY (Bruning et al., 2007; Choi et al., 2011). Our result was accordance with the reported result. Compared with rosiglitazone, four citrus flavonoids exhibited PPARY agonists. Bruning and his coworkers reported that BVT.13 and nTZDpa were part agonists of PPAR $\gamma$. They could contact the surface area of H3, making different hydrogen bonds with H3, as well as making more hydrophobic contacts overall. Both structures made several contacts with Arg 288, Ser 342 found on H3 (Bruning et al., 2007). Four tested citrus flavonoids exhibited hydrogen-bond interaction with $\mathrm{H} 3$ residues. Therefore, citrus flavonoids belong to part agonists.

Ligands docking to DPP IV protein active sites were shown in Figure 5. Figure 5A showed sitagliptin formed H-bonds with residues Glu 205, Glu 206, Tyr 662, Ser 209. Figure $5 B$ exhibited the strong interaction between hesperidin and DPP IV protein residues Glu 205, Ser 209, Cys 551, Lys 554, Tyr 585, Trp 629, Tyr 631, Tyr 662. Figure 5C illustrated the naringin formed H-bonds with protein residues Val 546, Ser 209, Tyr 662, Trp 629, Glu 206, Arg 125, Lys 554. Figure 5D depicted the interaction between neohesperidin and protein active sites. Figure 5E illustrated that nobiletin exhibited weak interaction with DPP IV protein via residues Trp 629, Ser 630, Tyr 631. The DPP IV resultant binding energy, $K_{i}, \mathrm{H}$-bond and other interaction with the active site residues are given below in the Table IV.

Parmar et al. indicated that sitagliptin exhibited good DPP IV inhibitory activity might be due to the its chemistry structure contains more electronegative groups F and N (Parmar et al., 2012). Hesperidin, naringin, neohesperidin obtained higher docking scores than sitagliptin. Interestingly, these three citrus flavonoids formed more hydrogen-bonds with DPP IV protein than sitagliptin. However, nobiletin formed only three Hydrogen-bonds with DPP IV protein and obtained the highest docking scores. These results indicated that the chemistry structure of citrus flavonoids might be contribute to the inhibitory activity of DPP IV. Hesperidin, naringin, neohesperidin have plenty of $\mathrm{OH}$ functional groups in the structures, which might promote the formation of $\mathrm{H}$-bonds between flavonoids and protein residues.

\section{Conclusion}

Docking studies of the ligand citrus flavonoids with four different target proteins showed that citrus flavonoids are good molecules which dock well with various targets related to diabetes mellitus. Therefore, citrus flavonoids play important roles in blood glucose regulation, might via activation of $\mathrm{GK}$ and $\mathrm{PPAR} \gamma$, whereas inhibition of GSK-3 $\beta$ and DPP IV.

\section{Acknowledgments}

This work was supported by "the Fundamental Research Funds for the Central Universities", and partially supported by Shanghai Leading Academic Discipline Project (B505), the National Special Fund for State Key Laboratory of Bioreactor Engineering (2060204).

\section{References}

Abu-Hamdah R, Rabiee A, Meneilly GS, Shannon RP, Andersen DK, Elahi D. The extra-pancreatic effects of glucagon-like peptide-1 and related peptides. J Clin Endocrinol Metab. 2009; 94: 1843-52.

Akhtar M, Bharatam PV. 3D-QSAR and molecular docking studies on 3-anilino-4-arylmaleimide derivatives as glycogen synthase kinase- $3 \beta$ inhibitors. Chem Biol Drug Design. 2012; 79: 560-71.

Akiyama S, Katsumata S, Suzuki K, Nakaya Y, Ishimi Y, Uehara M. Hypoglycemic and hypolipidemic effects of hesperidin and cyclodextrin-clathrate hesperetin in GotoKakizaki rats with type 2 diabetes. Biosci Biotechnol Biochem. 2009; 73: 2779-82.

Akiyama S, Katsumata S, Suzuki K, Ishimi Y, Wu J, Uehara M. Dietary hesperidin exerts hypoglycemic and hypolipidemic effects in streptozotocin-induced marginal type 1 diabetic rats. J Clin Biochem Nutr. 2010; 46: 87-92.

Baggio LL, Drucker DJ. Biology of incretins: GLP-1 and GIP. Gastroenterology 2007; 132: 2131-57.

Balamurugan R, Stalin A, Ignacimuthu S. Molecular docking of $\gamma$-sitosterol with some targets related to diabetes. Eur J Med Chem. 2012; 47: 38-43.

Benavente-Garcia O, Castillo J. Update on uses and properties of citrus flavonoids: New findings in anti-cancer, cardiovascular, and anti-inflammatory activity. J Agric Food. 2008; 2: 6185-205.

Bruning JB, Chalmers MJ, Prasad S, Busby SA, Kamenecka TM, He Y, Nettles KW, Griffin PR. Partial agonists activate PPARgamma using a helix 12 independent mechanism. Structure 2007; 15: 1258-71.

Chandramohan C, Ignacimuthu S, Pugalendi KV. A novel compound from Casearia esculenta (Roxb.) root and its effect on carbohydrate metabolism in streptozotocin-diabetic rats. Eur J Pharmacol. 2008; 590: 437-43.

Choi EJ, Ahn WS. Neuroprotective effects of chronic hesperetin administration in mice. Arch Pharm Res. 2008; 31: $1457-62$.

Choi JH, Banks AS, Kamenecka TM et al. Antidiabetic actions of a non-agonist PPARY ligand blocking Cdk5-mediated phosphorylation. Nature 2011; 477: 477-81.

Deacon CF. Therapeutic strategies based on glucagon-like peptide 1. Diabetes 2004; 53: 2181-89. 
Drucker DJ, Nauck MA. The incretin system: glucagon-like peptide-1 receptor agonists and dipeptidyl peptidase-4 inhibitors in type 2 diabetes. Lancet 2006. 368: 1696-705.

Johnson JL, Rupasinghe SG, Stefani, F, Schuler MA, Gonzalez de Mejia E. Citrus flavonoids luteolin, apigenin, and quercetin inhibit glycogen synthase kinase-3 $\beta$ enzymatic activity by lowering the interaction energy within the binding cavity. J Med Food. 2011; 14: 325-33.

Jung UJ, Lee MK, Jeong KS, Choi MS. The hypoglycemic effects of hesperidin and naringin are partly mediated by hepatic glucose-regulating enzymes in C57BL/KsJ-db/db mice. J Nutri. 2004; 4: 2499-503.

Jung UJ, Lee MK, Park YB, Kang MA, Choi MS. Effect of citrus flavonoids on lipid metabolism and glucose-regulating enzyme mRNA levels in type-2 diabetic mice. Int J Biochem Cell Biol. 2006; 38: 1134-45.

Kamata K, Mitsuya M, Nishimura T, Eiki J, Nagata Y. Structural basis for allosteric regulation of the monomeric allosteric enzyme human glucokinase. Structure 2004; 12: 429-38.

Khanfar MA, Hill RA, Kaddoumi A, El Sayed KA. Discovery of novel GSK-3 $\beta$ inhibitors with potent in vitro and in vivo activities and excellent brain permeability using combined ligand- and structure-based virtual screening. J Med Chem. 2010; 53: 8534-45.

Lee YS, Cha BY, Saito K et al. Nobiletin improves hyperglycemia and insulin resistance in obese diabetic ob/ob mice. Biochem Pharmacol. 2010; 79: 1674-83.

Liu Q, Chen L, Hu L, Shen X. Small molecules from natural sources, targeting signaling pathways in diabetes. Biochim Biophys Acta. 2010; 1799: 854-65.

Maltarollo VG, Honório KM. Ligand- and structure-based drug design strategies and PPARס/a selectivity. Chem Biol Drug Design. 2012; 80: 533-44.

Manthey JA, Grohmann K, Guthrie N. Biological properties of citrus flavonoids pertaining to cancer and inflammation. Curr Med Chem. 2001; 8: 135-53.

Nolte RT, Wisely GB, Westin S, Cobb JE, lambert MH, Kurokawa R, Rosenfeld MG, Wilson TM, Glass CK, Milburn MV. Ligand binding and co-activator assembly of the peroxisome proliferator-activated receptor- $\gamma$. Nature 1998; 16: $137-43$.

Osguthorpe DJ, Sherman W, Hagler AT. Generation of receptor structural ensembles for virtual screening using binding site shape analysis and clustering. Chem Biol Drug Des. 2012; 80: 182-93.

Osolodkin DI, Palyulin VA, Zefirov NS. Structure-based virtual screening of glycogen synthase kinase $3 \beta$ inhibitors: Analysis of scoring functions applied to large true actives and decoy sets. Chem Biol Drug Des. 2011; 78: 378-90.

Parmar HS, Jain P, Chauhan DS, Bhinchar MK, Munjal V, Yusuf M, Choube K, Tawani A, Tiwari V, Manivannan E, Kumar A. DPP-IV inhibitory potential of naringin: An in silico, in vitro and in vivo study. Diabetes Res Clin Pract. 2012; 97: 105-11.

Shen $\mathrm{W}, \mathrm{Xu} \mathrm{Y,} \mathrm{Lu} \mathrm{YH.} \mathrm{Inhibitory} \mathrm{effects} \mathrm{of} \mathrm{citrus} \mathrm{flavonoids} \mathrm{on}$ starch digestion and antihyperglycemic effects in HepG2 cells. J Agric Food Chem. 2012; 60: 9609-19.

Stoffel M, Froguel PH, Takeda J, Zouali H, el al. Human glucokinase gene: Isolation, characterization, and identification of two missense mutations linked to earlyonset non-insulin-dependent (type 2) diabetes mellitus. Proc Natl Acad Sci. 1992; 89: 7698-702.

Stumvoll M, Goldstein BJ, van Haeften TW. Type 2 diabetes: Principles of pathogenesis and therapy. Lancet 2005; 365: 1333-46.

Verspohl EJ. Novel therapeutics for type 2 diabetes: Incretin hormone mimetics (glucagon-like peptide-1 receptor agonists) and dipeptidyl peptidase-4 inhibitors. Pharmacol Ther. 2009; 124: 113-38.

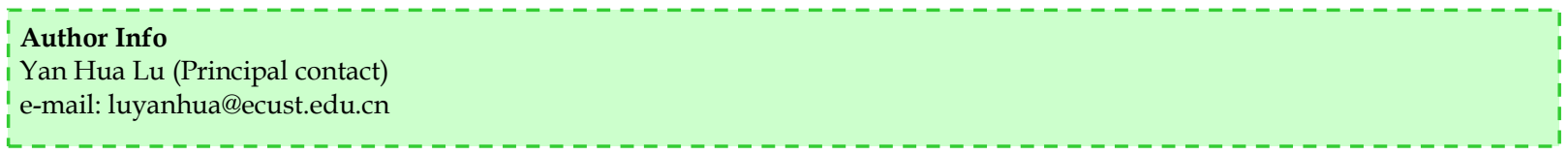

